\title{
CD8+ T-cell alveolitis in familial pulmonary alveolar microlithiasis
}

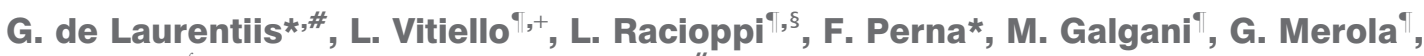 \\ P. Carratù ${ }^{f}$, M. Maniscalco*, S. Marsico ${ }^{\#}$ and M. Sofia*
}

ABSTRACT: Pulmonary alveolar microlithiasis (PAM) is a rare diffuse lung disease characterised by the accumulation of calcium phosphate microliths within the alveoli.

The causative mechanism of PAM has only recently been discovered, and involves a gene mutation of sodium phosphate co-transporter, which is expressed by alveolar epithelial cells. This mutation may have variable consequences on the clinical phenotype. However, pulmonary cell immune phenotyping in familial PAM has not previously been assessed.

In the present article, the analysis of bronchoalveolar lavage fluid of two siblings with PAM diagnosis revealed a pattern of lymphocytic alveolitis with accumulation of CD8+ T-cells. The clonal complexity of this lymphocyte's population was assayed by spectratyping, which showed an oligoclonal accumulation of T-cells with a restricted variable beta T-cell receptor (TCR) gene usage. TCR analysis in peripheral blood lymphocytes revealed no abnormal patterns of Tlymphocytes.

In the pulmonary alveolar microlithiasis familial cases reported, CD8-mediated maladaptive immune response may have taken place in the bronchoalveolar compartment. The relationship between this immune dysregulation and genetic background in pulmonary alveolar microlithiasis warrants further investigation.

KEYWORDS: Bronchoalveolar lavage, diffuse lung disease, pulmonary alveolar microlithiasis, TCD8 lymphocytes, T-receptor repertoire

P ulmonary alveolar microlithiasis (PAM) is a rare diffuse lung disease characterised by progressive intra-alveolar formation and accumulation of tiny, rounded corpuscles called microliths [1]. The analysis of reported cases reveals that the disease is prevalent amongst family units with a high rate of consanguinity among the parents of affected individuals [2,3], suggesting the hypothesis of the role of genetic factors in causing PAM [4]. Recently, CORUT et al. [5] have identified the gene mutation responsible for the disease as SLC34A2 (the type IIb sodium phosphate co-transporter gene), which is involved in phosphate homeostasis in several organs, including the lung. Due to this important discovery, it is now clear that intra-alveolar microliths' formation is the result of phosphate-chelating calcium present in the extracellular fluid. This is due to the loss of function of the gene product, expressed by alveolar II type epithelial cells, with a consequently decreased phosphate cell uptake needed for surfactant production. The consequence of this genetic background on phenotype is unknown.

Remarkably, despite its application in disease diagnosis, only few data exist documenting the phenotypic constituents of bronchoalveolar lavage fluid (BALF) in PAM; moreover, the Tlymphocyte antigen-recognising structure, T-cell receptor (TCR), has not been examined in PAM. TCRs are heterodimers comprising either $\alpha / \beta$ or $\gamma / \delta$ chains, each encoded by rearranged gene segments during T-cell ontogeny [6]. In particular, $\beta$-chain diversity is crucial for the ability of the T-cell population to recognise and respond to the vast number of different antigens that may be encountered [7]. Indeed, an accumulation of Tcells showing restricted TCR gene usage has been demonstrated in other diffuse lung diseases, including berillyosis, sarcoidosis and extrinsic allergic alveolitis [8-10]. The present authors report herein the BALF and peripheral blood lymphocyte (PBL) analysis of two siblings affected by familial PAM.

\section{AFFILIATIONS}

*Depts of Respiratory Medicine, AO Monaldi and

"Cellular and Molecular Biology and Pathology,

${ }^{\S}$ Center for Basic and Clinical Immunology (CISI), University Federico II of Naples, and, "Dept of Respiratory Medicine, AO Monaldi, Second University of Naples, Naples, +Laboratory of Immunobiology of Cardiovascular Disease, Dept of Medical Sciences and Rehabilitation IRCCS San Raffaele Pisana, Rome

${ }^{f}$ Dept of Respiratory Medicine, University of Bari, Bari, Italy.

CORRESPONDENCE

G. de Laurentiis

Via L. Bianchi

80131

Naples

Italy

Fax: 390815453213

Received:

November 062006

Accepted after revision:

January 082007

STATEMENT OF INTEREST

None declared. 


\section{PATIENTS AND METHODS}

\section{Patients}

The present study subjects comprised two siblings, a 24-yr-old male and a 27-yr-old female, who had received a diagnosis of PAM in childhood by observation of typical radiological features and from the finding of calcospherites in BALF. Both patients had no referred smoking history. At the visit, they denied significant respiratory symptoms except for mild exercise limitation. Radiography and high-resolution computed tomography (HRCT) features showed the typical findings of PAM (fig. 1) [11]. The patients gave their written informed consent to take part in the study, which was approved by the local Ethical Committee.
The whole-body bone scintigraphic scan, by intravenous administration of $740 \mathrm{MBq}$ of technetium 99m-methylene diphosphonate $\left({ }^{99 \mathrm{~m}} \mathrm{Tc}-\mathrm{MDP}\right)$, showed both patients to have an intensive bone remodelling activity with a diffuse and abnormal pulmonary uptake in all the scan planes of the thoracic region. The laboratory data, including urine analysis, sweat chloride, blood glucose, calcium and phosphate blood levels, renal and liver function tests, and thyroid hormone and uric acid blood levels were within normal ranges in both patients. The tuberculin Mantoux skin test was negative. Microbiological examination for Mycobacteria, fungi and bacterial species did not reveal any alveolar contamination. BALF samples from both patients were examined by PCR
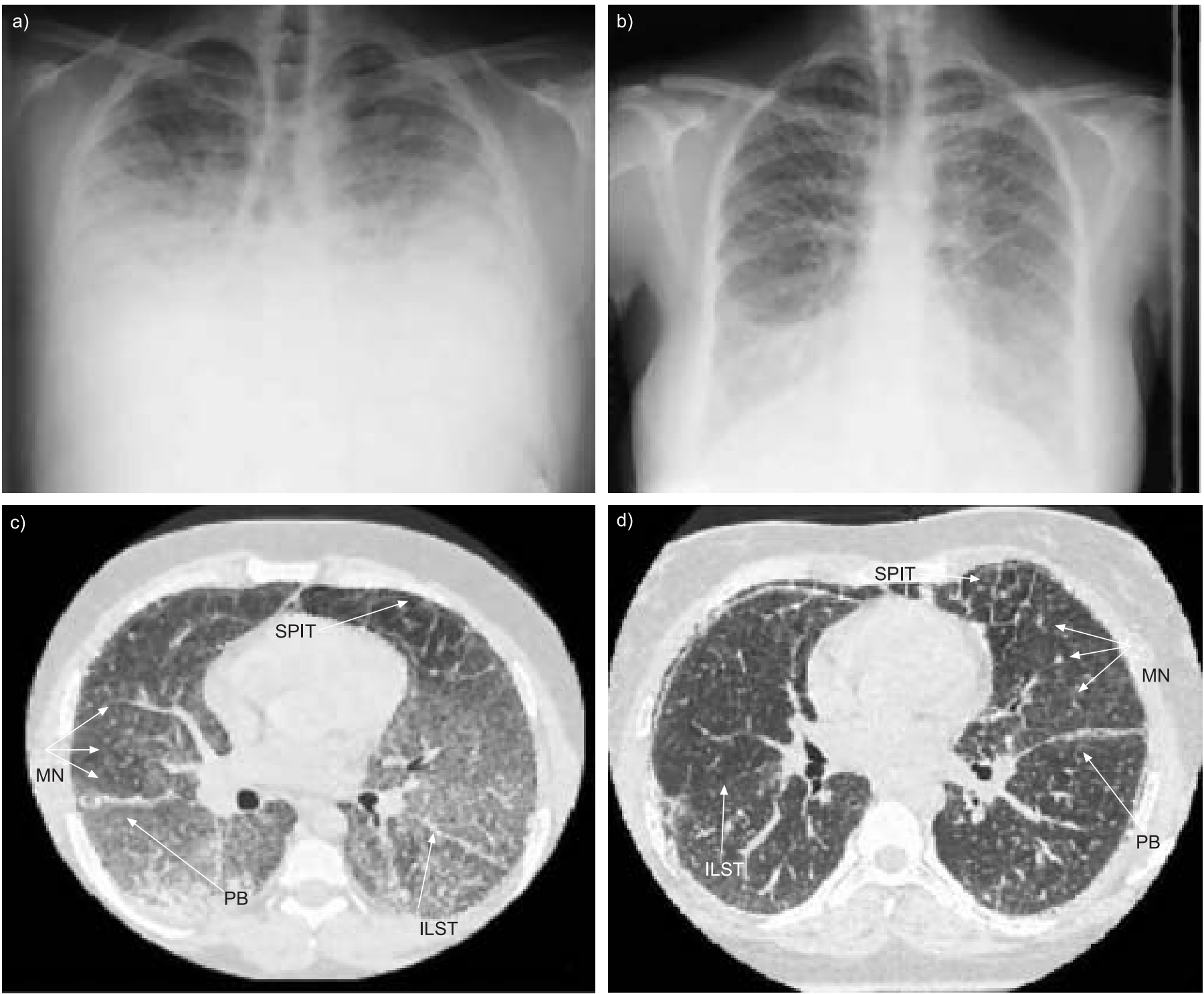

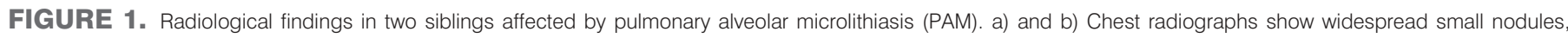

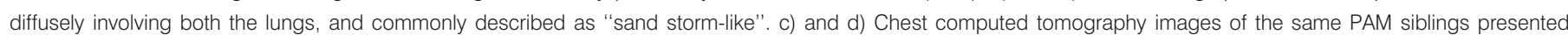

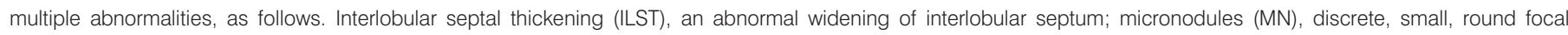

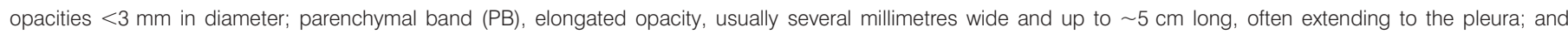
subpleural interstitial thickening (SPIT), consisting of thickened fissures. 
analysis for detection of Herpes virus type 1 and 2, EpsteinBarr virus and cytomegalovirus; all results were negative.

\section{Genealogical tree}

The genealogical tree of PAM patients is represented in figure 2. A consanguinity degree between the parents of the patients was shown, as they were sons of two cousins. In the familial line of the mother, multiple cases of renal disorders were reported, including nephrolithiasis, polycystic kidney disease and chronic renal failure. In the father's familial line, there was an high rate of chronic liver disease complicated by cirrhosis in 10 members (II3, III2, III3, III18, III19, IV2, IV3, IV4, IV5 and IV12) or liver carcinoma in a further three cases. At the time of the present study, the other female sibling of the two patients was unaffected, and reported no respiratory, renal or liver diseases.

\section{Bronchoalveolar lavage procedures and handling of cells}

Bronchoalveolar lavage (BAL) was performed with a flexible bronchoscope with local xylocaine anaesthesia. The bronchoscope was wedged in a bronchus in the middle lobe and sterile phosphate-buffered saline solution at $37^{\circ} \mathrm{C}$ was instilled in three $50-\mathrm{mL}$ aliquots. After each instillation, the fluid was gently aspirated and collected in a siliconised plastic bottle coded by number and placed on ice. BALF was filtered through two layers of sterile cotton gauze to remove mucus and was then centrifuged at $240 \times g$ for $10 \mathrm{~min}$. BALs were carried out on the first hospital admission (time 0) and then 3 months later (time 1), when the clinical state and respiratory symptoms were steady. Total alveolar cell population was counted using an optical microscope with a Burker chamber. Recovered cells showed $>90 \%$ viability, as assayed by trypan blue exclusion. Smears for differential counts were prepared by cytocentrifugation, followed by May-Grunwald-Giemsa staining. Peripheral blood mononuclear cells (PBMC) were separated from heparinised blood by Ficoll-Hypaque gradient centrifugation (Pharmacia, Uppsala, Sweden), washed twice and then diluted in RPMI-1640 (Sigma Aldrich, Milan, Italy).

\section{Immunofluorescence and flow cytometry}

Monoclonal antibodies (cluster of differentiation (CD)45 PC5, CD3 FITC, CD4 PE, CD8 PC5, CD19 PE, CD16 PE; Beckman Coulter Co., Marseille, France) were utilised according to the following triple sampling: CD45 PC5, CD3 FITC, CD19 PE (immunological gate on CD45-positive cells in order to evaluate T- and B-lymphocytes); CD45 PC5, CD3 FITC, CD16 $\mathrm{PE}$ (immunological gate on CD45-positive cells in order to evaluate T- and natural killer-lymphocytes); and CD3 FITC, CD4 PE, CD8 PC5 (immunological gate on CD3-positive cells in order to evaluate CD4+ and CD8+ T-lymphocytes). Aliquots of BAL and PBMC cells were washed twice in RPMI-1640 (Sigma Aldrich, Milan, Italy), triple stained with the reported antibodies and, finally, analysed in a Coulter Epics XL (Beckman Coulter Co.) with an XL System II software version 3.0 according to a previously reported procedure [12].

\section{Analysis of beta variable CDR3 etherogeneity length by spectratyping}

The beta variable (BV) region of the TCR was studied using the spectratyping method as previously reported [13]. T-cells were separated from BALF and from PBL into CD8+ and CD8- cells by magnetic sorting with coated beads (Dynabeads; Dynal, Oslo, Norway). RNA was prepared using Trizol (Invitrogen, Milan, Italy) according to the manufacturer's instructions. Reverse transcription and PCR amplification for the 26 different BV TCR families was performed in a single-step

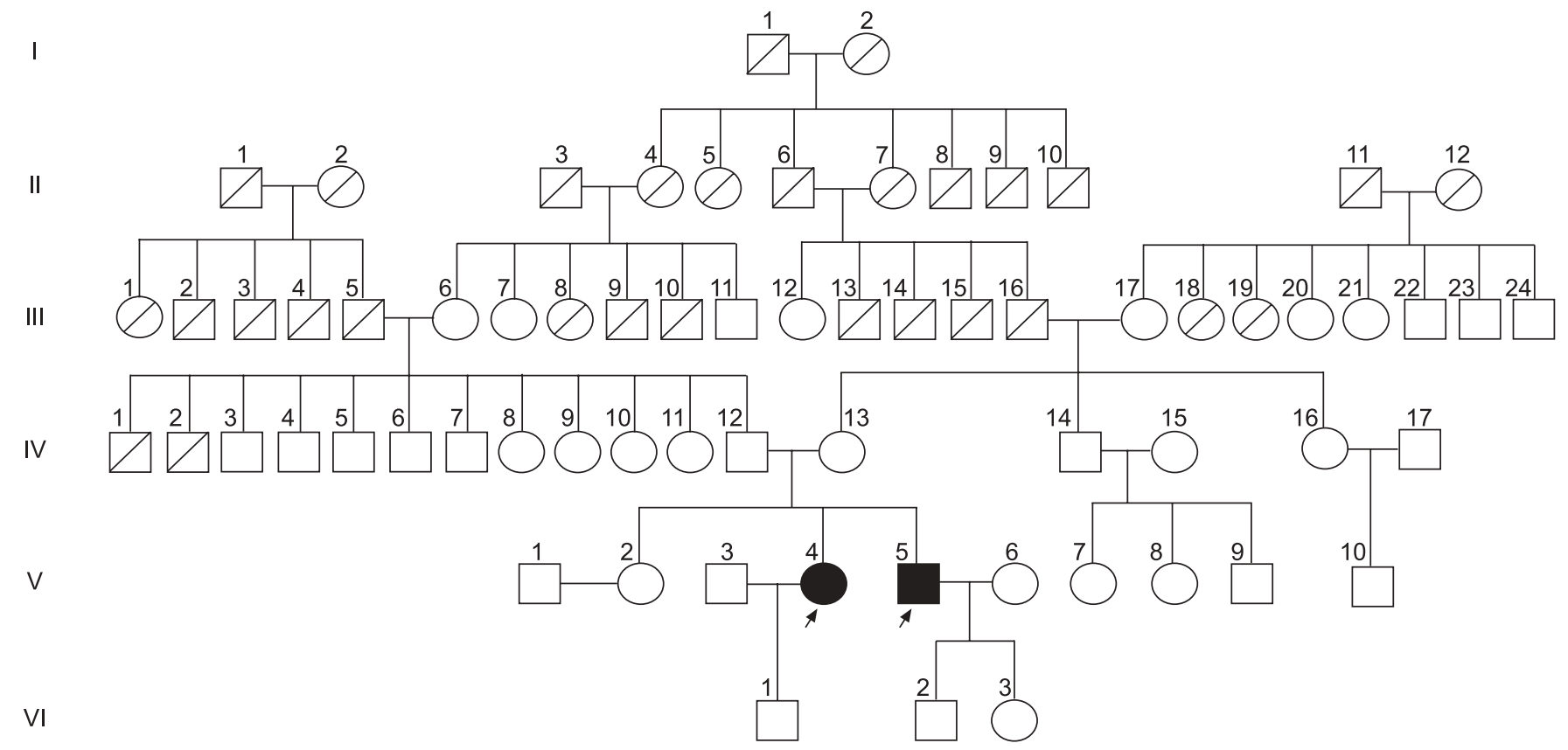

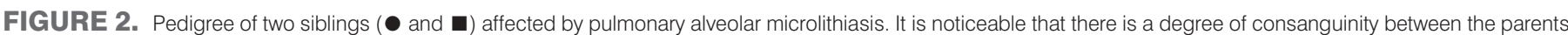

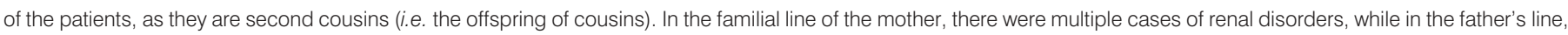
many siblings were affected by chronic hepatitis complicated by cirrhosis or liver carcinoma. $\square$ : male; $\bigcirc$ : female; /: indicates the subject died 
reaction with the SuperScript $\mathrm{TM}$ III One-Step RT-PCR System with Platinum ${ }^{\circledR}$ Taq DNA Polymerase (Invitrogen). Briefly, the same primers were used both for reverse transcription and for amplification. A common reverse primer (CB3 primer) was used in conjunction with 24 different forward primers (TCRBV gene family primers), whose sequences corresponded to those described by GORSKI et al. [14]. To resolve the composition of each BV TCR family, the PCR products were run on a sequencing gel in a fluorescence-based DNA sequencer (ABI 377; Applied Biosystems, Warrington, UK). Normally, each TCR family is resolved by this technique as a series of bands with a Gaussian distribution. Each alteration, in either the distribution or the intensity of the single bands, represents a perturbation in the given BV TCR family reflecting the involvement of that family in an immune response towards one or more antigens.

\section{RESULTS}

\section{Clinical parameters}

Lung volumes were within normal limits, while the diffusing capacity of the lung for carbon monoxide $(D \mathrm{~L}, \mathrm{CO})$ showed mild-to-moderate reduction in both subjects (DL,CO $8.72(74 \%)$ and $6.47(66.5 \%), D \mathrm{~L}, \mathrm{CO}$ per unit of alveolar volume 1.32 $(77.5 \%)$ and $1.55(84 \%)$ in patients 1 and 2, respectively). Resting blood gases revealed mild hypoxaemia compared with age (8.49 and $9.25 \mathrm{kPa}$, respectively).

\section{BALF and PBMC}

The total number of cells recovered by both BALF (at time 0 and time 1), the percentage of lymphocytes in BALF and the relative occurrence of major lymphocyte subsets in BALF and PBL lymphocytes of both PAM patients are reported in table 1. The total BALF cellular count was raised $\left(3.0 / 7.0 \times 10^{5}\right.$ and $5.0 / 4.0 \times 10^{5}$ cells $\cdot \mathrm{mL}^{-1}$ at time 0 and time 1 , respectively) compared with normal values for a person of the same age $\left(0.5-1 \times 10^{5}\right.$ cells $\cdot \mathrm{mL}^{-1}$, as previously reported [12]). Lymphocyte percentage, determined by differential counting of cells on the alveolar cytogram using May-Grunwald-Giemsa stained smears, was largely increased in both patients at the different times, and always consisted of $>30 \%$ BALF T-cell subpopulations, as analysed by flow cytometry. This revealed that, in both patients, an increased amount of the CD8+/CD3+ subset was present, with subsequent inversion of $\mathrm{CD} 4+/ \mathrm{CD} 8+$ ratio. The PBL cellular pattern did not show any lymphocytic abnormalities compared with the reference values of subjects of same age.

\section{TCR BV gene usage}

To evaluate the clonal complexity of the lymphocyte population accumulating in the bronchoalveolar compartment of the present study's PAM patients, the CDR3 heterogeneity length of $26 \mathrm{BV}$ genes expressed by T-lymphocytes isolated from BALF was measured by spectratyping analysis at time 1 of the study. As CD8+ T-cells represented the large majority of Tlymphocytes in both patients, this subset was separated by magnetically labelled antibodies in order to analyse specifically the TCR repertoire expressed by cytotoxic CD8+ T-cells. The current authors found a restricted BV gene usage in both patients. In patient 1, 14 out of $26 \mathrm{BV}$ families were expressed in CD8+ lymphocytes (fig. 3). In CD8+ cells, only four families displayed a Gaussian profile, and BV25 alone represented 31\% of the whole BV repertoire expressed in CD8+. Spectratyping analysis revealed a marked restriction in BV usage and also in lymphocytes isolated from the BALF of patient 2 . Thirteen out of $26 \mathrm{BV}$ families were expressed in CD8+ T-cells. None of them showed a normal profile. Noticeably, BV2 represented $36 \%$ of whole BV families expressed in CD8+ lymphocytes (fig. 3). It is noteworthy that, in both patients, BV6.2 and BV7 showed a similar pattern and a comparable frequency of expression. Furthermore, BV2, 5.1, 7, 8, 13, 17, 18, 20 and 21 are present in CD8 lymphocytes of both patients. Conversely, spectratyping analysis of PBLs revealed no differences between PAM patients and healthy volunteers of the same age (fig. 3 and data not shown).

\begin{tabular}{|c|c|c|c|c|}
\hline & Time 0 & Time 1 & Time 0 & Time 1 \\
\hline \multicolumn{5}{|l|}{ BAL } \\
\hline Cells $n \times 10^{6}$ & 13.5 & 40.5 & 22.5 & 18 \\
\hline Lymphocytes \% & 35 & 40 & 55 & 46 \\
\hline CD3+ \% & 97 & 93 & 94 & 95 \\
\hline $\mathrm{CD} 3+/ \mathrm{CD} 4+\%$ & 16 & 21 & 22 & 12 \\
\hline CD3+/CD8+ \% & 80 & 50 & 60 & 76 \\
\hline \multicolumn{5}{|l|}{ PBL } \\
\hline
\end{tabular}




\section{DISCUSSION}

In the two siblings studied, the diagnosis of PAM was based on clinical history, typical chest radiological appearance (radiography and HRCT), and on the presence of calcosperites in BALF, the latter being considered diagnostic for PAM (fig. 4). Furthermore, the present patients showed no signs of remote or current alveolar infection, which may induce PAM-like alterations. Accordingly, RATJEN et al. [15] reported an unusual case of PAM, which was associated with intense lymphocytic alveolitis resulting from lymphocytic interstitial pneumonitis (LIP). LIP was considered unlikely in the present subjects from the clinical and radiological features. Furthermore, there was no sign of any underlying systemic or autoimmune disorders, which are frequently associated with LIP, and all systemic auto-antibody tests were negative. Moreover, the subjects did not present with HIV or Epstein-Barr virus infection and had no evidence of other viral infections.

To evaluate the reliability and the reproducibility of the data, BALF and blood analysis were repeated in both patients at a different time (3 months later).

The present results show that the BALF of two siblings affected by familial PAM present an intense lymphocytic alveolitis, characterised by the prevalence of CD8+ T-cells and associated with abnormalities in their TCR pattern. In all the BALF, flow cytometry revealed an unexpected accumulation of CD8+
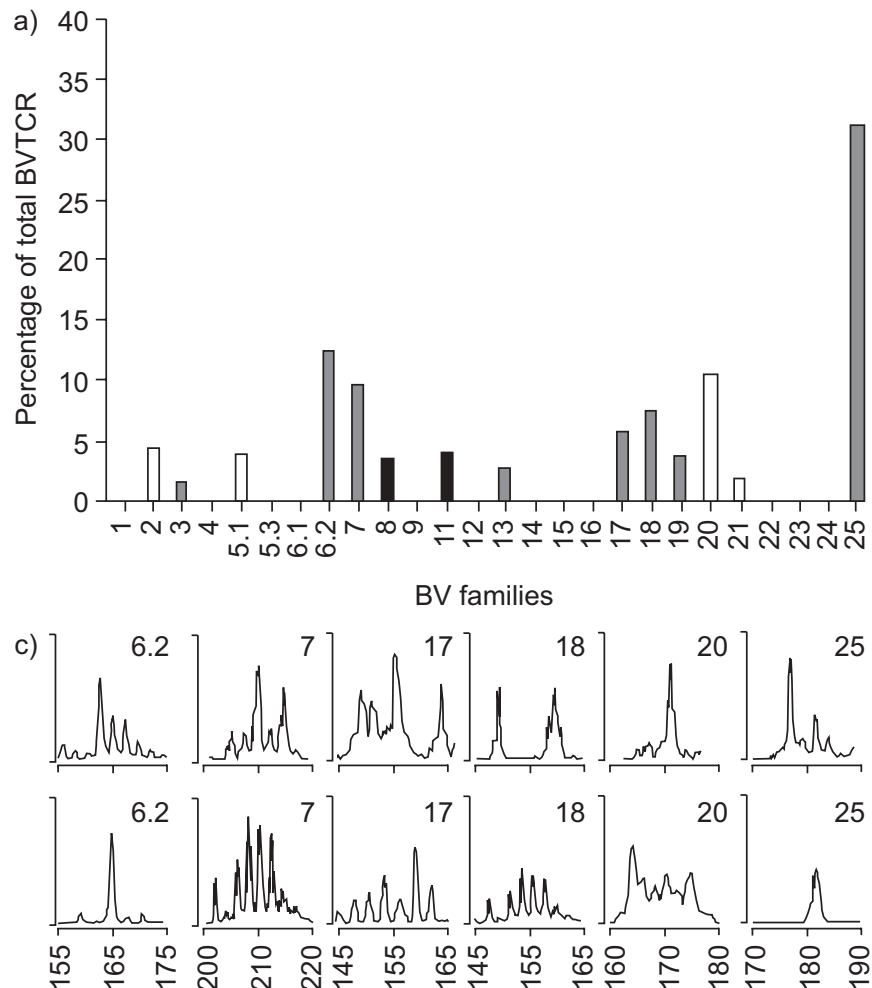

T-cells that, at the spectratyping analysis carried out at time 1 of the study, expressed a restricted BV gene usage. Some of the clonal expansions found in the BALF T-lymphocytes' TCR repertoire showed a similar profile in both PAM patients (families 6.2 and 7). To the present authors' knowledge, a formal immunophenotyping analysis of BALF in familial PAM is not available in the literature. TCR repertoire can be defined as normal when all BV families are expressed at comparable frequencies, and show a bell-shaped "Gaussian" profile. Altered profiles, such as those found in the BALF lymphocytes of the present study's patients, may reflect either an antigendriven clonal selection or an intrinsic process of immune dysregulation taking place in the alveolar space; indeed, the majority of BV families expressed were altered and only one BV family (BV25 and BV2 in patient 1 and 2, respectively) represented $>30 \%$ of the TCR expressed by the CD8+ T-cells population. The absence of the same alteration in PBLs indicates that the perturbation is not intrinsic to the CD8+ Tlymphocytes, but is specific for BALF T-lymphocytes.

There are also some similarities between the BALF pattern shown in PAM in the present study and what has been reported in the subacute phase of hypersensitivity pneumonitis (HP) [16]. Actually, a CD8+ T-lymphocyte alveolitis with accumulation of T-cells using a limited number of BV genes has been documented in HP $[10,17,18]$. However, the history and clinical course of the present cases was not suggestive of

b)

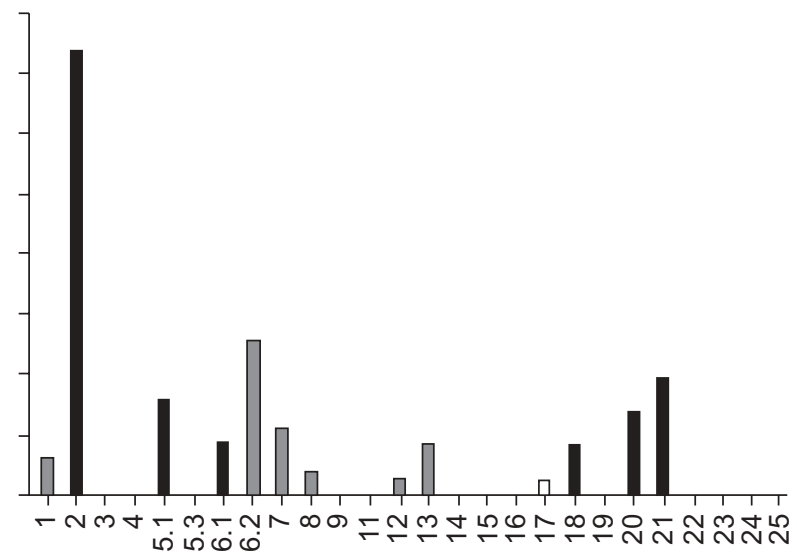

d)
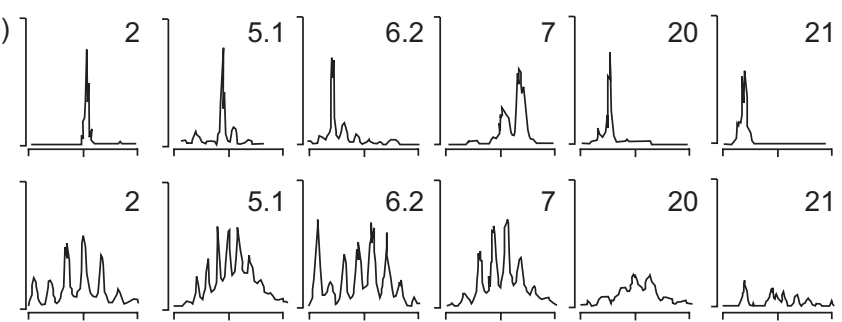

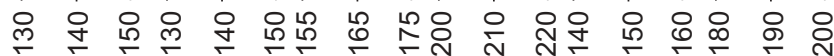

FIGURE 3. Spectratyping analysis of CD8+ T-lymphocytes in bronchoalveolar lavage fluid. Frequency distribution of beta variable (BV) families expressed by CD8+Tlymphocytes in bronchoalveolar lavage fluid (BALF) from a) patient 1 and b) patient 2 . $\square$ : normal families ( $>5$ rearrangements distributed in a Gaussian fashion); $\square$ : skewed families ( $<5$ rearrangements); $\mathbf{a}$ : oligomonoclonal families (1-2 major peaks). c) and d) CDR3 size distribution profiles of BV families over-expressed in BALF from c) patient 1 and d) patient 2. The top panels represent expression from BALF and the bottom panels represent expression in peripheral blood lymphocytes. The number in each box indicates the BV family. The expression profile of correspondent BV families in peripheral blood is shown. Families are aligned by molecular size. TCR: T-cell receptor. 


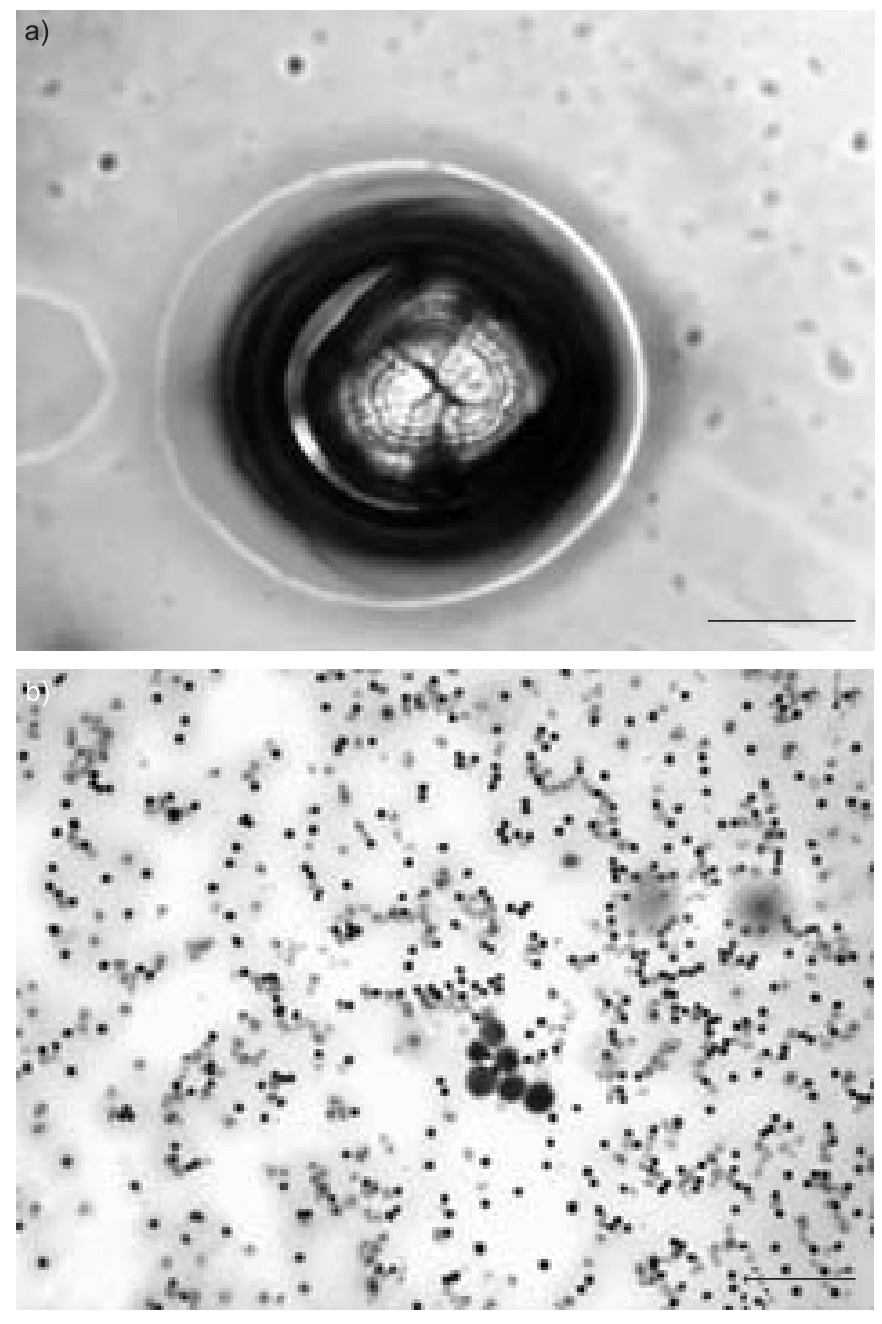

FIGURE 4. Microscopic images of pulmonary alveolar microlithiasis (PAM). a) Typical rounded and tiny intra-alveolar microliths (May-Grunwald-Giemsa stained; scale bar $=10 \mu \mathrm{m})$. b) Accumulation of lymphocytes in bronchoalveolar lavage fluid of one of the two siblings affected by PAM, showing a feature of lymphocitic alveolitis. This finding was presented in both patients at different times (scale bar $=50 \mu \mathrm{m}$ ).

HP. Moreover, the present authors were unable to detect identical T-cell clones in the lung and in the blood of the PAM subjects, as recently reported in patients with HP.

Recently, CORUT et al. [5] identified the gene responsible for PAM in a large family and identified homozygous mutation in SLC34A2 (the type IIb sodium phosphate co-transporter gene). In light of this finding, it is certain that PAM is a recessively inherited disease showing a full genetic penetration, with environmental factors playing a minor role. However, in contrast with the predicted severe effects of the identified mutations on the protein product of the gene or on gene expression, the patients with PAM studied by CORUT et al. [5] had mild clinical phenotypes, with the exception of the smokers. This lack of a genotype-phenotype correlation was also supported by the variation in age at onset among the affected members of the large family evaluated.

As the identification of gene mutation responsible for PAM has only very recently become available, after the completion of the present article, the present authors did not examine DNA from their subjects for the SLC34A2 mutation; however, this genetic abnormality was documented by CORUT et al. [5] in all their unrelated subjects with PAM diagnosis and the present authors believe that this might have implications in the interpretation of their data. The type Ilb sodium phosphate co-transporter plays a crucial role in the homeostasis of inorganic phosphate and it is expressed in lung only in alveolar type II cells, which are responsible for production of surfactant, comprising phospholipids and surfactant proteins (SP), the most abundant of which are SP-A and SP-D [19]. Noticeably, alveolar lymphocytes are exposed to surfactant, which in turn may inhibit mitogen-induced lymphocyte proliferation [20, 21]. Furthermore, SP-D and SP-A may provide an important link between innate and adaptive immunity, by modulation of dendritic cells and T-cell functions [22]. Both SP-A and SP-D would suppress T-cell activation in the alveolar space, which if it occurred, could result in an inflammatory cascade with the possible consequence of lung damage and impaired gas exchange [23]. Thus, the current authors could speculate that in PAM, the altering of the alveolar environment by a genetic mutation affecting sodium phosphate co-transporter in surfactant-producing epithelial cells would determine some local imbalance in immune effector response.

Additionally, PAM is a recessive inherited disease, occurring with both a familial and sporadic pattern [5, 24, 25], and with a high proportion of familial cases among Turkish and Italian case series. Interestingly, examination of the genealogical tree in the present PAM cases showed an important consanguinity grade with a high frequency of chronic liver disease and hepatic cirrhosis in the paternal line where clonal T-cell abnormalities have been reported [26, 27].

In conclusion, in the present study's familial pulmonary alveolar microlithiasis cases, an intense lymphocytic alveolitis was found in the bronchoalveolar lavage fluid with the prevalence of CD8+ T-cells, associated with abnormalities in their T-cell receptor pattern. From the present study, the issue remains whether this is a hitherto unrecognised component of pulmonary alveolar microlithiasis or whether this is an idiosyncratic (or other) reaction limited to this particular pedigree. Additional studies are required to examine the relationship between abnormalities of bronchoalveolar lavage cells and the recently characterised genetic background in pulmonary alveolar microlithiasis, in order to understand the cellular and molecular mechanisms accounting for the tissue damage in this rare, currently untreatable diffuse lung disease [28].

\section{ACKNOWLEDGEMENTS}

The authors would like to thank C. Calabrese for the microscopy imaging support.

\section{REFERENCES}

1 Prakash UB. Pulmonary alveolar microlithiasis. Semin Respir Crit Care Med 2002; 23: 103-113.

2 Mariotta S, Ricci A, Papale M, et al. Pulmonary alveolar microlithiasis: report on 576 cases published in the literature. Sarcoidosis Vasc Diffuse Lung Dis 2004; 21: 173-181. 
3 Senyigit A, Yaramis A, Gurkan F, et al. Pulmonary alveolar microlithiasis: a rare familial inheritance with report of six cases in a family. Contribution of six new cases to the number of case reports in Turkey. Respiration 2001; 68: 204-209.

4 Lauta VM. Pulmonary alveolar microlithiasis: an overview of clinical and pathological features together with possible therapies. Respir Med 2003; 97: 1081-1085.

5 Corut A, Senyigit A, Ugur SA, et al. Mutations in SLC34A2 cause pulmonary alveolar microlithiasis and are possibly associated with testicular microlithiasis. Am J Hum Genet 2006; 79: 650-656.

6 Marrack P, Kappler J. The T-cell receptor. Science 1987; 238 1073-1076.

7 O'Brien DP, Baecher-Allan CM, Burns RP Jr, Shastri N, Barth RK. Elimination of T cell-receptor b-chain diversity in transgenic mice restricts antigen-specific but not alloreactive responses. Immunology 1997; 91: 375-382.

8 Fontenot AP, Kotzin BL, Comment CE, Newman LS. Expansions of T-cell subsets expressing particular T-cell receptor variable regions in chronic beryllium disease. Am J Respir Cell Mol Biol 1998; 18: 581-589.

9 Zissel G, Baumer I, Fleischer B, Schlaak M, MullerQuernheim J. TCR V beta families in T cell clones from sarcoid lung parenchyma, BAL, and blood. Am J Respir Crit Care Med 1997; 156: 1593-1600.

10 Wahlstrom J, Berlin M, Lundgren R, et al. Lung and blood $\mathrm{T}$-cell receptor repertoire in extrinsic allergic alveolitis. Eur Respir J 1997; 10: 772-779.

11 Deniz O, Ors F, Tozkoparan E, et al. High resolution computed tomographic features of pulmonary alveolar microlithiasis. Eur J Radiol 2005; 55: 452-460.

12 Mazzarella G, Bianco A, Perna F, et al. T lymphocyte phenotypic profile in lung segments affected by cavitary and non-cavitary tuberculosis. Clin Exp Immunol 2003; 132: 283-288.

13 Masci AM, Palmieri G, Vitiello L, et al. Clonal expansion of $\mathrm{CD}^{+}$BV8 $\mathrm{T}$ lymphocytes in bone marrow characterizes thymoma-associated B lymphopenia. Blood 2003; 101: 3106-3108.

14 Gorski J, Yassai M, Zhu X, Kissela B, Keever C, Flomenberg N. Circulating $T$ cell repertoire complexity in normal individuals and bone marrow recipients analyzed by CDR3 size spectratyping. Correlation with immune status. I Immunol 1994; 152: 5109-5119.

15 Ratjen FA, Schoenfeld B, Wiesemann HG. Pulmonary alveolar microlithiasis and lymphocytic interstitial pneumonitis in a ten year old girl. Eur Respir J 1992; 5: 1283-1285.
16 Agostini C, Trentin L, Facco M, Semenzato G. New aspects of hypersensitivity pneumonitis. Curr Opin Pulmon Med 2004; 10: 378-382.

17 Facco M, Trentin L, Nicolardi L, et al. T cells in the lung of patients with hypersensitivity pneumonitis accumulate in a clonal manner. J Leukoc Biol 2004; 75: 798-804.

18 Raulf M, Liebers V, Steppert C, Baur X. Increased $\gamma / \delta$ positive T-cells in blood and bronchoalveolar lavage of patients with sarcoidosis and hypersensitivity pneumonitis. Eur Respir J 1994; 7: 140-147.

19 Wright JR. Immunomodulatory functions of surfactant. Physiol Rev 1997; 77: 931.

20 Kaltreider HB, Salmon SE. Immunology of the lower respiratory tract: functional properties of bronchoalveolar lymphocytes obtained from the normal canine lung. J Clin Invest 1973; 52: 2211.

21 Wilsher ML, Hughes DA, Haslam PL. Immunoregulatory properties of pulmonary surfactant: influence of variations in the phospholipid profile. Clin Exp Immunol 1988; 73: 117.

22 Hussain S. Role of surfactant protein A in the innate host defense and autoimmunity. Autoimmunity 2004; 37: 125-130.

23 Borron PJ, Mostaghel EA, Doyle C, Walsh ES, McHeyzerWilliams MG, Wright JR. Pulmonary surfactant proteins A and D directly suppress CD3+/CD4+ cell function: evidence for two shared mechanisms. I Immunol 2002; 169: 5844-5850.

24 Mariotta S, Guidi L, Papale M, Ricci A, Bisetti A. Pulmonary alveolar microlithiasis: review of Italian cases. Eur J Epidemiol 1997; 13: 587-590.

25 Moran CA, Hochholzer L, Hasleton PS, Johnson FB, Koss MN. Pulmonary alveolar microlithiasis. A clinicopathologic and chemical analysis of seven cases. Arch Pathol Lab Med 1997; 121: 607-611.

26 Zekri AR, Sabry GM, Bahnassy AA, Shalaby KA, AbdelWahabh SA, Zakaria S. Mismatch repair genes (hMLH1, hPMS1, hPMS2, GTBP/hMSH6, hMSH2) in the pathogenesis of hepatocellular carcinoma. World J Gastroenterol 2005 28, 11: 3020-3026.

27 Rushbrook SM, Ward SM, Unitt E, et al. Regulatory T cells suppress in vitro proliferation of virus-specific CD8+ T cells during persistent hepatitis C virus infection. J Virol 2005; 79: 7852-7859.

28 Poletti V, Costabel U, Casoni GL, Bigliazzi C, Drent M, Olivieri D. Rare infiltrative lung diseases: a challenge for clinicians. Respiration 2004; 71: 431-443. 